\title{
On Conformally Flat Stationary Axisymmetric Spacetimes
}

\author{
Alberto A. García and Cuauhtemoc Campuzano \\ Departamento de Física. \\ Centro de Investigación y de Estudios Avanzados del IPN. \\ Apdo. Postal 14-740, 07000 México DF, MEXICO
}

\begin{abstract}
It is shown that within conformally flat stationary axisymmetric spacetimes, besides of the static family, there exists a new class of metrics, which is always stationary and axisymmetric. All these spacetimes, the static and the stationary ones, are endowed with an arbitrary function depending on the two non-Killingian coordinates. The explicit form of this function can be determined once the coupled matter, i.e., the energy-momentum tensor is given. One might hope possible extensions of this result to black holes on two-branes in four dimensions.
\end{abstract}

PACS numbers: 04.20.Jb, 02.40.H,M

\section{INTRODUCTION}

In 1976, Collinson [1] formulated the following theorem: Every conformally flat stationary axisymmetric space-time is necessarely static. If the source of such space-time is a perfect fluid, then the space-time metric can be reduced to the usual Schwarzschild interior metric. The main goal of present work is to establish the existence of a new class of intrinsically stationary axisymmetric spacetimes, which, at the same time, are conformally flat. Hence, the Collinson theorem fails to be true in its first statement. In what follows the complex coefficients associated to the conformal Weyl tensor for the general stationary axisymmetric metric are given. Next, in Sec. II the general integrals for conformally flat spaces are presented. Sec. III deals with conformally flat locally static spaces. As a by-product, we demonstrate here that one can isolate spacetimes of the form $R \times B T Z$ [2, 3]; the results of 22 suggest the possibility of constructing four dimensional black holes bound to two-branes. In Sec. IV the general expression of conformally flat stationary axisymmetric metrics is given. Finally, some concluding remarks are stated.

The starting point in our study is the stationary axisymmetric line-element

$$
\begin{aligned}
d s^{2} & =e^{-2 Q(z, \bar{z})} d z d \bar{z} \\
& +\frac{e^{-2 G(z, \bar{z})}}{a+b}[a(z, \bar{z}) d \sigma+d \tau][b(z, \bar{z}) d \sigma-d \tau]
\end{aligned}
$$

where $\partial_{\sigma}$ and $\partial_{\tau}$ are killing vectors, such that one is spacelike and the second is a timelike one.

The evaluation of the Newman-Penrose curvature coefficients- Weyl complex components- with respect to the null tetrad basis,

$$
g=2 e^{1} \otimes e^{2}-2 e^{3} \otimes e^{4}
$$

*Electronic address: gas@fis.cinvestav.mx where

$$
\begin{aligned}
& e^{1}=\frac{1}{\sqrt{2}} e^{-Q} d z, \quad e^{3}=\frac{1}{\sqrt{2}} \frac{e^{-G}}{\sqrt{a+b}}(b d \phi-d t), \\
& e^{2}=\frac{1}{\sqrt{2}} e^{-Q} d \bar{z}, \quad e^{4}=\frac{1}{\sqrt{2}} \frac{e^{-G}}{\sqrt{a+b}}(a d \phi+d t),
\end{aligned}
$$

yields the following nonvanishing components

$$
\begin{aligned}
\Psi_{0} & =\frac{e^{2 Q}}{a+b}\left[2 \frac{\partial a}{\partial z} \frac{\partial P}{\partial z}+\frac{\partial^{2} a}{\partial z^{2}}-\frac{2}{a+b}\left(\frac{\partial a}{\partial z}\right)^{2}\right], \\
\bar{\Psi}_{4} & =\frac{e^{2 Q}}{a+b}\left[2 \frac{\partial b}{\partial z} \frac{\partial P}{\partial z}+\frac{\partial^{2} b}{\partial z^{2}}-\frac{2}{a+b}\left(\frac{\partial b}{\partial z}\right)^{2}\right], \\
6 \Psi_{2} & =\frac{e^{2 Q}}{(a+b)^{2}}\left[2(a+b)^{2} \frac{\partial^{2} P}{\partial z \partial \bar{z}}+5 \frac{\partial a}{\partial z} \frac{\partial b}{\partial \bar{z}}-\frac{\partial a}{\partial \bar{z}} \frac{\partial b}{\partial z}\right],
\end{aligned}
$$

where $P=P(z, \bar{z}):=Q-G$.

\section{CONFORMALLY FLAT STATIONARY AXISYMMETRIC SPACETIMES; GENERAL CASE $\partial a / \partial z \neq \partial b / \partial z \neq 0$}

If one demands the spacetime to be conformally flat, then the Weyl tensor has to vanish, which is equivalent to fulfill the requirements $\Psi_{0}=\Psi_{4}=\Psi_{2}=0$. Accordingly, one has:

$$
\Psi_{0}=0 \Longrightarrow 2 \frac{\partial a}{\partial z} \frac{\partial P}{\partial z}+\frac{\partial^{2} a}{\partial z^{2}}-\frac{2}{a+b}\left(\frac{\partial a}{\partial z}\right)^{2}=0
$$

and,

$$
\Psi_{4}=0 \Longrightarrow 2 \frac{\partial b}{\partial z} \frac{\partial P}{\partial z}+\frac{\partial^{2} b}{\partial z^{2}}-\frac{2}{a+b}\left(\frac{\partial b}{\partial z}\right)^{2}=0 .
$$

Subtracting equations (5)-(6), one obtains

$$
\frac{\partial}{\partial z}\left[\frac{e^{2 P}}{(a+b)^{2}}\left(\frac{\partial a}{\partial z}-\frac{\partial b}{\partial z}\right)\right]=0,
$$


therefore its integration yields

$$
\frac{\partial a}{\partial z}-\frac{\partial b}{\partial z}=\bar{g}(\bar{z})(a+b)^{2} e^{-2 P} .
$$

Next, dividing Eq. (5) by $\partial a / \partial z$ and Eq. (6) by $\partial b / \partial z$, and subsequently adding the resulting equations, one gets

$$
\frac{\partial}{\partial z} \ln \left[\frac{e^{4 P}}{(a+b)^{2}} \frac{\partial a}{\partial z} \frac{\partial b}{\partial z}\right]=0
$$

thus

$$
\frac{\partial a}{\partial z} \frac{\partial b}{\partial z}=\bar{h}(\bar{z})(a+b)^{2} e^{-4 P}
$$

For real $\Psi_{2}$, one arrives at the condition

$$
\frac{\partial a}{\partial z} \frac{\partial b}{\partial \bar{z}}=\frac{\partial a}{\partial \bar{z}} \frac{\partial b}{\partial z}
$$

Since the functions $a, b$, and $P$ are real, therefore from Eq. (7) one has

$$
g(z) \frac{\partial}{\partial z}(a-b)=\bar{g}(\bar{z}) \frac{\partial}{\partial \bar{z}}(a-b) .
$$

By introducing a new variable $z$, such that $g(z) \frac{\partial}{\partial z} \rightarrow \frac{\partial}{\partial z}$, the above equation becomes

$$
\left(\frac{\partial}{\partial z}-\frac{\partial}{\partial \bar{z}}\right)(a-b)=0
$$

This transformation, allowed because of the freedom in the choice of the variable $z$, is equivalent to set $g(z)=1$. Hence $a-b=F(z+\bar{z})$. Introducing the real coordinates $x$ and $y$ through $z=x+i y$, denoting with dots the derivatives with respect to $x$, and substituting $a=b+$ $F(x)$ into Eq. (9), one obtains

$$
\dot{F}\left(\frac{\partial}{\partial z}-\frac{\partial}{\partial \bar{z}}\right) b=0
$$

If $\dot{F}=0$, then using linear transformations of the Killingian variables $\tau$ and $\sigma$, one can achieve $a=b$, and consequently the metric is static.

\section{A. Metric for $\dot{F} \neq 0, b=b(z+\bar{z})$ and $a=a(z+\bar{z})$}

If now $\dot{F} \neq 0$, then $b=b(x)$ and $a=a(x)$. Without loss of generality, equations (7) and (8) rewrite correspondingly

$$
\dot{a}-\dot{b}=(a+b)^{2} e^{-2 P}
$$

and

$$
\dot{a} \dot{b}=\epsilon k^{2}(a+b)^{2} e^{-4 P},
$$

where the parameter $\epsilon= \pm 1$. Introducing new dependent functions $X=X(x)$ and $Y=Y(x)$ on the variable $x$ according with

$$
\begin{aligned}
& a+b=2 k Y, a=k(Y+X), \\
& a-b=2 k X, b=k(Y-X),
\end{aligned}
$$

the Eq. (13) becomes

$$
\dot{X}=\frac{d X}{d x}=2 k Y^{2} e^{-2 P} \rightarrow d x=\frac{d X}{2 k Y^{2}} e^{2 P},
$$

while Eq. (14) amounts to

$$
\dot{Y}^{2}-\dot{X}^{2}=4 k^{2} \epsilon Y^{2} e^{-4 P} .
$$

Substituting $e^{-2 P}$ from Eq. (16) into Eq. (17) one obtains

$$
\dot{Y}^{2}-\dot{X}^{2}=\epsilon \frac{\dot{X}^{2}}{Y^{2}}
$$

or equivalently, dividing by $\dot{X} \neq 0$, one has

$$
\left(\frac{d Y}{d X}\right)^{2}=1+\frac{\epsilon}{Y^{2}} \rightarrow \frac{d Y}{d X}=\nu \frac{\sqrt{Y^{2}+\epsilon}}{Y}, \nu= \pm 1
$$

with general integral

$$
\nu\left(X-X_{0}\right)=\sqrt{Y^{2}+\epsilon}, \rightarrow Y^{2}=\left(X-X_{0}\right)^{2}-\epsilon,
$$

where $X_{0}$ is an integration constant.

It remains to integrate the equation arising from $\Psi_{2}=$ 0 ,

$$
\begin{aligned}
6 \Psi_{2} & =\frac{e^{2 Q}}{(a+b)^{2}}\left[(a+b)^{2} P_{, x, x}+4 a_{, x} b_{, x}\right] \\
& =\frac{e^{2 Q}}{Y^{2}}\left[2 Y^{2} P_{, x, x}+\left(Y_{, x}\right)^{2}-\left(X_{, x}\right)^{2}\right] .
\end{aligned}
$$

Using instead of $x$ the new variable $X, \frac{d}{d x}=\dot{X} \frac{d}{d X}, \dot{P}=$ $\dot{X} P_{, X}=: \dot{X} P^{\prime}$, taking into account Eq. (18), one gets

$$
6 \Psi_{2}=\frac{e^{2 Q}}{Y^{2}} \dot{X}^{2}\left[2 Y^{2}\left(P^{\prime \prime}+\frac{\ddot{X}}{\dot{X}^{2}} P^{\prime}\right)+\frac{\epsilon}{Y^{2}}\right] .
$$

Therefore, for vanishing $\Psi_{2}$, one has

$$
P^{\prime \prime}+\frac{\ddot{X}}{\dot{X}^{2}} P^{\prime}+\frac{\epsilon}{2 Y^{4}}=0 .
$$

Differentiation of $\dot{X}$ from Eq. 16 with respect to $x$, yields

$$
\ddot{X}=4 k \dot{X}\left[Y Y^{\prime}-Y^{2} P^{\prime}\right] e^{-2 P}=2 \dot{X}^{2}\left(Y^{\prime} / Y-P^{\prime}\right) .
$$

Replacing $\ddot{X}$ from the above relation into Eq. (23), one arrives at

$$
P^{\prime \prime}-2 P^{\prime 2}+2 \frac{Y^{\prime}}{Y} P^{\prime}+\frac{\epsilon}{2 Y^{4}}=0 .
$$

Introducing the function $K=\exp (-2 P)$, the Eq. 25) becomes

$$
K^{\prime \prime}+2 \frac{Y^{\prime}}{Y} K^{\prime}-\frac{\epsilon}{Y^{4}} K=0 .
$$


Substituting in this equation $Y^{\prime}$ from Eq. (19), and $Y$ from Eq. (20) in terms of $X$, considering that $\nu^{2}=1$, one has

$$
\begin{aligned}
& {\left[\left(X-X_{0}\right)^{2}-\epsilon\right]^{2} K^{\prime \prime} } \\
+ & 2\left[\left(X-X_{0}\right)^{2}-\epsilon\right]\left(X-X_{0}\right) K^{\prime}-\epsilon K=0 .
\end{aligned}
$$

To obtain the general solution of Eq. (27), one accomplishes the change $K(X)=M(X)\left[\left(X-X_{0}\right)^{2}-\epsilon\right]^{-1 / 2}$, which yields

$$
M^{\prime \prime}=0 \rightarrow M(X)=C_{0}+C_{1} X .
$$

Therefore

$$
\begin{aligned}
e^{-2 P}=K(X) & =\left(C_{0}+C_{1} X\right)\left[\left(X-X_{0}\right)^{2}-\epsilon\right]^{-1 / 2} \\
& =\left(C_{0}+C_{1} X\right) / Y .
\end{aligned}
$$

In terms of the new coordinates $X$, after trivial scaling and coordinate translations: $2 k y \rightarrow y, X-X_{0} \rightarrow$ $X, \sqrt{2}\left(\tau+k X_{0} \sigma\right) \rightarrow T, \sqrt{2} k \sigma \rightarrow \Phi$, the studied metric amounts to

$$
\begin{aligned}
d s^{2} & =e^{-2 G(X, y)} \frac{1}{\sqrt{X^{2}-\epsilon}}\left[\frac{d X^{2}}{\left(C_{0}+C_{1} X\right)\left(X^{2}-\epsilon\right)}\right. \\
& \left.+\left(C_{0}+C_{1} X\right) d y^{2}+k\left(-\epsilon d \Phi^{2}-2 X d \Phi d T-d T^{2}\right)\right] .
\end{aligned}
$$

To establish whether this metric could be diagonalized or not, one accomplishes additionally further linear transformations in the metric-sector

$$
d \Sigma^{2}:=k\left(-\epsilon d \Phi^{2}-2 X d \Phi d T-d T^{2}\right)
$$

of the Killingian variables $T$ and $\Phi$ of the form

$$
\begin{aligned}
& d T=\alpha d \tau^{\prime}+\beta d \sigma^{\prime}, \\
& d \Phi=\gamma d \tau^{\prime}+\delta d \sigma^{\prime}, \quad \alpha \delta-\beta \gamma \neq 0,
\end{aligned}
$$

for real $\alpha, \beta, \gamma$, and $\delta$. The $g_{\tau^{\prime} \sigma^{\prime}}$ component of the metricsector $d \Sigma^{2}$ amounts to

$$
g_{\tau^{\prime} \sigma^{\prime}}=-k[\epsilon \gamma \delta+X(\beta \gamma+\alpha \delta)+\alpha \beta]
$$

thus, $g_{\tau^{\prime} \sigma^{\prime}}$ may vanish is there exists a real solution of the equations

$$
\begin{aligned}
& \beta \gamma+\alpha \delta=0, \\
& \alpha \beta+\epsilon \gamma \delta=0 .
\end{aligned}
$$

The general solution of this system is given by

$$
\begin{aligned}
& \alpha= \pm \gamma \sqrt{\epsilon}, \gamma=\gamma, \\
& \beta=\mp \delta \sqrt{\epsilon}, \delta=\delta,
\end{aligned}
$$

therefore these constants are real parameters only in the case $\epsilon=1$. Accordingly, the metric-sector components $g_{\tau^{\prime} \tau^{\prime}}$ and $g_{\sigma^{\prime} \sigma^{\prime}}$ acquire the form

$$
g_{\tau^{\prime} \tau^{\prime}}=-2 k \gamma^{2}(\epsilon \pm \sqrt{\epsilon} X), \quad g_{\sigma^{\prime} \sigma^{\prime}}=-2 k \delta^{2}(\epsilon \mp \sqrt{\epsilon} X),(36)
$$

where the choice of the upper (lower) sign in $g_{\tau^{\prime} \tau^{\prime}}$, has to be accompanied by the choice of the upper (lower) sign in $g_{\sigma^{\prime} \sigma^{\prime}}$.

Only for the branch of metrics with $\epsilon=1$ one can carry out real linear transformations of the Killingian coordinates such that the metric-sector $d \Sigma^{2}$ becomes diagonal, which on its turn implies the diagonal character of the whole metric $d s^{2}$.

The case $\epsilon=-1$ deserves special attention; the transformations (32) are pure imaginary ones, and the corresponding metric tensor components become complex, fact which is forbidden in real Einsteinian Relativity.

\section{METRIC FOR CONFORMALLY FLAT LOCALLY STATIC SPACETIMES, $\epsilon=1$}

For $\epsilon=1$-the only case in which conformally flat stationary axisymmetric spacetimes can be locally diagonalized- with an additional scaling transformation of the form $\sqrt{2} \gamma \tau^{\prime} \rightarrow \tau$ and $\sqrt{2} \delta \sigma^{\prime} \rightarrow \sigma,(X \rightarrow x)$, the corresponding metric becomes

$$
\begin{aligned}
d s^{2} & =\frac{e^{-2 G(x, y)}}{\sqrt{x^{2}-1}}\left[\frac{d x^{2}}{\left(C_{0}+C_{1} x\right)\left(x^{2}-1\right)}+\left(C_{0}+C_{1} x\right) d y^{2}\right. \\
& \left.-(1 \mp x) d \sigma^{2}-(1 \pm x) d \tau^{2}\right] .
\end{aligned}
$$

This branch corresponds to the choice $k=1$; the case $k=$ -1 yields similar final results. Considering the Killingian metric sector

$$
d \Sigma^{2}:=-(1+x) d \sigma^{2}-(1-x) d \tau^{2},
$$

one faced out two possibilities:

A: $x<-1, \partial_{\tau}$ timelike Killing vector, and $\partial_{\sigma}$ spacelike Killing vector,

B: $x>1, \partial_{\tau}$ spacelike Killing vector, and $\partial_{\sigma}$ timelike Killing vector.

Case A: Introducing a new coordinate $A$,

$$
A^{2}=-\frac{1+x}{1-x}, 1+x<0, x=-\frac{A^{2}+1}{1-A^{2}}, 1-A^{2}>0,
$$

identifying $\sigma \rightarrow \phi, \tau \rightarrow t, C_{0}-C_{1} \rightarrow 2 \alpha, C_{0}+C_{1} \rightarrow-2 \beta$, and $G(x, y)-\frac{1}{2} \ln \left(\alpha+\beta A^{2}\right) / A \rightarrow G(A, y)$, one arrives at the expression

$$
\begin{aligned}
d s^{2}=e^{-2 G(A, y)} & {\left[\frac{d A^{2}}{\left(\alpha+\beta A^{2}\right)^{2}}+d y^{2}\right.} \\
+ & \left.\frac{A^{2}}{\alpha+\beta A^{2}} d \phi^{2}-\frac{d t^{2}}{\alpha+\beta A^{2}}\right],
\end{aligned}
$$

Case B: Similarly as we treated the previous case, one introduces a new variable $A$, namely

$$
A^{2}=\frac{x-1}{x+1}, x-1>0, x=\frac{1+A^{2}}{1-A^{2}}, 1-A^{2}>0,
$$


identifying $\sigma \rightarrow t, \tau \rightarrow \phi, C_{0}+C_{1} \rightarrow 2 \alpha, C_{1}-C_{0} \rightarrow 2 \beta$, and $G(x, y)-\frac{1}{2} \ln \left(\alpha+\beta A^{2}\right) / A \rightarrow G(A, y)$, one arrives at the expression (40).

\section{A. Metric for Conformally Flat Locally Static Spacetimes, $\epsilon=1$, a $R \times B T Z$ representation}

We would like to point out an alternative representation of the above metric when a negative cosmological constant is present, $\lambda \sim 1 / l^{2}$.

By accomplishing in metric (30), with $\epsilon=1, k=1$, a $\mathrm{SL}(2, \mathrm{R})$-transformation of the Killingian coordinates given by

$$
\begin{aligned}
& d T=\alpha d t+\beta d \phi, \\
& d \Phi=\gamma d t+\delta d \phi, \quad \alpha \delta-\beta \gamma \neq 0,
\end{aligned}
$$

where

$$
\begin{aligned}
& \alpha=-\frac{1}{\sqrt{2 l}}\left(b_{0} r_{+}+a_{0} r_{-}\right), \quad \beta=\frac{1}{\sqrt{2}}\left(a_{0} r_{+}+b_{0} r_{-}\right) \\
& \gamma=\frac{1}{\sqrt{2 l}}\left(b_{0} r_{+}-a_{0} r_{-}\right), \quad \delta=\frac{1}{\sqrt{2}}\left(a_{0} r_{+}-b_{0} r_{-}\right) \\
& r_{ \pm}=\sqrt{\frac{l}{2}} \sqrt{M l \pm \sqrt{M^{2} l^{2}-J^{2}}}
\end{aligned}
$$

with constants $C_{0}$ and $C_{1}$, appearing Eq.(30), fulfilling the relations

$$
C_{0}+C_{1}=-2 a_{0}^{2}, C_{0}-C_{1}=-2 b_{0}^{2},
$$

together with a transformation of the $\mathrm{x}-$ coordinate, $X \rightarrow$ $x$,

$$
x=\frac{b_{0}^{2}\left(r^{2}-r_{-}^{2}\right)+a_{0}^{2}\left(r^{2}-r_{+}^{2}\right)}{b_{0}^{2}\left(r^{2}-r_{-}^{2}\right)-a_{0}^{2}\left(r^{2}-r_{+}^{2}\right)}
$$

where $l$ is related with the cosmological constant, $M$, and $J$ stand correspondingly for the global mass and the total angular momentum of the BTZ solution, one arrives at a $R \times B T Z$ representation of the studied metric, namely

$$
\begin{aligned}
d s^{2} & =e^{-2 G(r, y)}\left(d y^{2}+d s_{B T Z}^{2}\right) \\
& =e^{-2 G(r, y)}\left[d y^{2}+\frac{d r^{2}}{\frac{J^{2}}{4 r^{2}}-M+\frac{r^{2}}{l^{2}}}\right. \\
& \left.+r^{2}\left(d \phi-\frac{J}{2 r^{2}} d t\right)^{2}-\left(\frac{J^{2}}{4 r^{2}}-M+\frac{r^{2}}{l^{2}}\right) d t^{2}\right]
\end{aligned}
$$

\section{GENERAL METRIC FOR CONFORMALLY FLAT STATIONARY AXISYMMETRIC SPACETIMES}

From the metric (30) when $\epsilon=-1$, one arrives at a new result: there is no way to carry out a diagonalization of the whole metric $d s^{2}$, it remains locally stationary axisymmetric. This conclusion contradicts the theorem by Collinson, which asserts that" Every conformally flat stationary axisymmetric spacetime is necessarily static". Restoring the $\{x, y, \phi, t\}$-typing in metric (30); for positive $k: \sqrt{k} T \rightarrow t, \sqrt{k} \Phi \rightarrow \phi$, while for $k$ negative, $k=-\kappa: \sqrt{\kappa} T \rightarrow \phi, \sqrt{\kappa} \Phi \rightarrow-t$, one arrives at the canonical form of conformally flat stationary axisymmetric spacetimes

$$
\begin{aligned}
d s^{2} & =\frac{e^{-2 G(x, y)}}{\sqrt{x^{2}+1}}\left[\frac{d x^{2}}{\left(C_{0}+C_{1} x\right)\left(x^{2}+1\right)}+\left(C_{0}+C_{1} x\right) d y^{2}\right. \\
& \left.+\left(x^{2}+1\right) d \phi^{2}-(d t+x d \phi)^{2}\right]
\end{aligned}
$$

Since we started with a general form for the metric of stationary axisymmetric spacetimes, and arrived at the above expression for this metric in the case of conformal flatness through the determination of the general solution of the zero-Weyl tensor equations, the above metric is the more general form for conformally flat stationary axisymmetric spacetimes. Of course, one can give other representations of this metric by using coordinate transformations of the variable $x$ and $y$; for instance representations in terms of trigonometric or hyperbolic functions. The expression of the factor-function $G(x, y)$ depends on the matter-field content, i.e., on the energy-momentum tensor for different kinds of fields in the Einstein equations; different energy-tensors will give rise to different $G(x, y)$.

\section{CONCLUSIONS}

On the light of the present results, we conclude that the Collinson theorem is wrong. There exist branches of spacetimes which are conformally flat and at the same time are stationary and axisymmetric. The conformal factor of this class of metrics depends on non-Killingian variables $x$ and $y$; its explicit expression depend on the sources of the Einstein equations.

\section{Acknowledgments}

The authors thank E. Ayón-Beato for useful discussions. This work has been partially supported by CONACyT Grant 38495E. 
[1] C.D. Collison, Gen. Rel. Grav. 7 (1976) 419.

[2] Roberto Emparan, Gary T. Horowitz and Robert C. Myers, JHEP 01 (2000) 007.

[3] A. A. García, "Exact Solutions and Scalar Fields in Gra- vity: Recent Developments" Kluwer Academic /Plenum Publishers, New York, 2001. 